\title{
Acromioclavicular Dislocation Management Using Semitendinosus Tendon Graft: Report of 2 cases
}

\author{
NS Dhaniwala ${ }^{1 *}$, Aashay Kekatpure ${ }^{2}$, Rameez Bukhari ${ }^{3}$ and Mukund N Dhaniwala ${ }^{4}$ \\ ${ }^{1}$ Professor of Orthopedics, Department of Orthopedics, JNMC and AVBRH, Datta Meghe Institute of Medical Sciences, Sawangi, Wardha, India \\ ${ }^{2}$ Associate Professor of Orthopedics, Department of Orthopedics, JNMC and AVBRH, Datta Meghe Institute of Medical Sciences, Sawangi, \\ Wardha, India \\ ${ }^{3}$ Resident Orthopedics, Department of Orthopedics, JNMC and AVBRH, Datta Meghe Institute of Medical Sciences, Sawangi, Wardha, India \\ ${ }^{4}$ Fellow in Orthopedics, Lokmanya Tilak Marg, Pune, India
}

*Corresponding Author: NS Dhaniwala, Professor of Orthopedics, Department of Orthopedics, JNMC and AVBRH, Datta Meghe Institute of Medical Sciences, Sawangi, Wardha, India.

Received: July 16, 2019; Published: August 26, 2019

\begin{abstract}
Acromioclavicular joint dislocation is a common injury seen in adults due to fall on the point of shoulder. Its nonoperative treatment is rarely successful from cosmesis and restoration of anatomy point of view. Various surgical methods with inconsistent results from cosmetic and function point of view have been described [1]. Autologous semitendinosus graft and or synthetic sutures are being used to reconstruct coracoclavicular ligament with or without acromioclavicular ligament recently with more success [2]. The present report describes short term anatomic results of reconstruction of coracoclavicular ligament using semitendinosus tendon graft from the ipsilateral thigh of the patient in two patients having right sided AC joint dislocation (type iii injury).
\end{abstract}

Keywords: Tendon Graft; Acromioclavicular Joint; Injury

\section{Introduction}

Acromioclavicular joint (ACJ) is a synovial joint between the lateral end of clavicle and medial part of acromion process of scapula. It contains an articular cartilage disc arising from its capsule. The integrity of AC joint depends on its capsule, acromioclavicular ligament and primarily on coracoclavicular ligament, having two parts (conoid ligament between coracoid process to the conoid tubercle of clavicle, and trapezoid ligament between coracoid process to trapezoid line of clavicle). AC joint dislocation is commonly classified in three types, depending on the clinical and radiological findings. Fracture of the lateral end of clavicle clinically behaves like AC joint injury; hence its management is on similar lines.
The coracoclavicular ligament anchors the distal clavicle to the coracoid process of scapula, and helps in all shoulder movements including scapulothoracic movement. This strong structure maintains the position of clavicle in relation to the acromion process and its rupture leads to sagging of scapula in turn causing prominence of the lateral end of the clavicle. Acromioclavicular injuries account for $9-12 \%$ of all shoulder injuries. Acromioclavicular joint injuries were first described by Tossey., et al. in type 1,2 and 3. Rockwood modified type 3 injuries and added type 4,5 and 6 to Tossey's classification [1]. Type 1 and type 2 are mild injuries involving sprain in type 1 and rupture of acromioclavicular ligament and acromioclavicular joint capsule in type 2 . These do not cause much anatomic 
disability or function loss as coracoclavicular ligament remains intact. Type 3 injuries consists of disruption of acromioclavicular and coracoclavicular ligaments, and the distal clavicle goes up by at least the thickness of the clavicle. In type 4 the clavicle goes posteriorly into or through trapezius muscle. Type 5 involves detachment of deltoid and trapezius muscle insertion from the distal half of clavicle besides ligaments' rupture. This may cause gross separation of AC joint. Type 6 injuries are rare and occur due to extreme abduction tearing the ligaments and forcing the clavicle beneath coracoid process. Repair of coracoclavicular and acromioclavicular ligaments along with joint capsule are difficult and less successful due to traumatic rupture and attrition. Reconstruction of these ligaments, primarily coracoclavicular ligament, is essential for restoring cosmesis, anatomy and strength of shoulder function. Various surgical treatment involving use of wire, transfer of coracoacromial ligament, suture anchors, suture loops, coracoclavicular screw fixation and reconstruction of acromioclavicular and coracoclavicular ligament using endobutton either in open surgery or arthroscopic surgery have been described [1-3]. Mazzocca., et al. [4] used autologous semitendinosus for coracoclavicular reconstruction successfully in acute dislocations. The present case reports also show satisfactory restoration of joint anatomy after reconstruction of coracoclavicular ligaments in one fresh and one 3 months old AC joint dislocation using autologous semitendinosus tendon.

\section{Case Report 1}

A 67 years old male village farmer reported in orthopedic OPD with the chief complaints of pain and restriction of right shoulder movement for 3 months. He gave a history of fall on his right shoulder after being hit by a bull, after which he developed pain immediately in the right shoulder region. Pain subsided gradually with drugs. A few days later he noticed difficulty in lifting his right shoulder up and doing day today chores in farm and hence reported to our center.

Examination revealed prominence of lateral end of right clavicle near acromion process with mild tenderness. Head of humerus was in normal articulation but the right scapula was at a lower level in comparison to the left as seen from back. Shoulder flexion was from 0 to 50 degrees, extension from 0 to 20 degrees, abduction from 0 to 40 degrees, internal rotation from 0 to 70 degrees and external rotation from 0 to 30 degrees. X-ray right shoulder AP view (Figure 1) showed displaced fracture at the tip of the lateral end of clavicle with small bone piece lying inferior to the clavicle lateral end which was seen more prominently. MRI examination of shoulder reported Osteoarthritic changes in the right acromioclavicular and glenohumeral joint with partial tear of subscapularis and supraspinatus tendon, with bicipital tendinopathy, besides the fracture at the lateral end of the right clavicle.

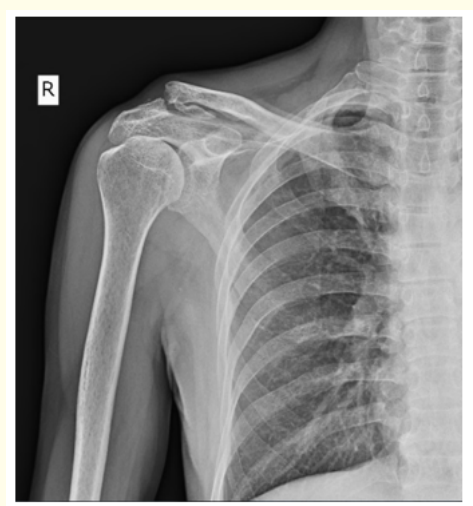

Figure 1: Shows fracture lateral tip of right clavicle with dislocation of $\mathrm{AC}$ joint.

The patient was planned for open reduction and coracoclavicular ligament reconstruction using semitendinosus tendon. In beach chair position after general anesthesia, right sided semitendinosus tendon graft of length $20 \mathrm{~cm}$. was harvested under tourniquet using standard technique. Right shoulder was approached by oblique incision extending from acromion process towards coracoid process. After splitting deltopectoral fascia bony landmarks were clearly delineated. A hole of diameter $4.5 \mathrm{~mm}$ was drilled in the clavicle after oblique osteotomy of lateral end of clavicle. Semitendinosus graft anchored properly using non-absorbable braided suture no.5 (Ethibond) was passed around the base of coracoid as a loop and then passed through the hole in the lateral end of the clavicle, thus reducing the prominence of the clavicle and elevating scapula level and was sutured on itself. The remaining length of the continuous tendon graft was used to reconstruct the acromioclavicular ligament, by bridging the gap across the acromion and the osteotomized clavicle end. A thin Kirschner wire was passed from acromion into the lateral end of the clavicle for further support (Figure 2). Closure was done in layers properly and arm chest strap applied. The patient's shoulder was kept immobilized for six weeks, after stitch 
removal at ten days and Kirschner wire removal at three weeks. Gradual active mobilization and active use of shoulder was encouraged afterwards. Post operatively, the anatomy of the shoulder was restored clinically and the x-ray showed improved acromioclavicular level. The patient continues to be under follow up with restored shoulder cosmesis and gradual gain in movement.

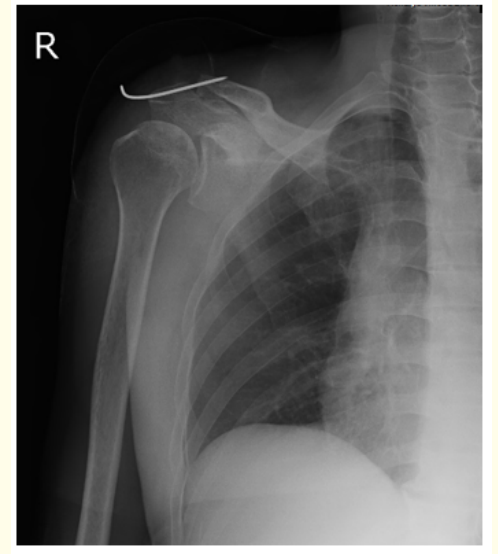

Figure 2: Shows Kirschner wire across AC joint.

\section{Case Report 2}

A 42 years old male was diagnosed to have acromioclavicular joint (AC joint) dislocation of the right-side following fall from motor bike on the affected shoulder one day back. His shoulder movements were painfully restricted. Examination demonstrated tenderness at $\mathrm{AC}$ joint with prominent lateral end of right clavicle. $\mathrm{X}$-ray showed dislocation of $\mathrm{AC}$ joint with acromion process at a lower level (Figure 3). The patient was managed by reconstruction of coracoclavicular ligament using semitendinosus tendon from right thigh, tied along its length in a zig-zag fashion with nonabsorbable suture no.5 (Ethibond), and reinforcement with Mersilene tape as a loop between coracoid process and osteotomized lateral end of clavicle. Remaining tendon graft was used to reconstruct acromioclavicular ligament also. The shoulder was immobilized for 6 weeks followed by gradual active stepped exercises and use of the limb. The case was followed up for more than 6 months, with anatomy restored effectively and movements near normal. Follow up x-ray at 02 weeks and 12 weeks (Figure 4 and Figure 5 respectively) show maintained AC joint level. The patient continues to be under follow up.

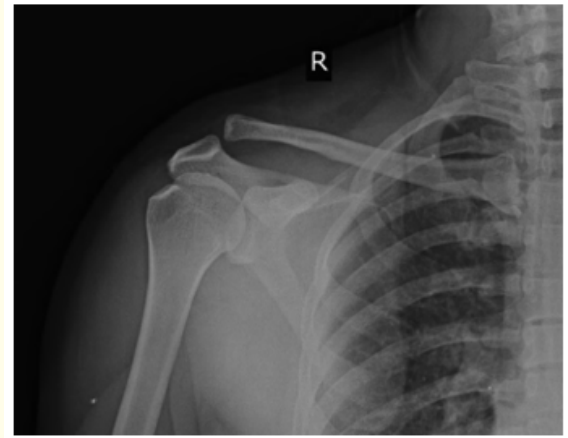

Figure 3: Shows AC joint dislocation, case 2.

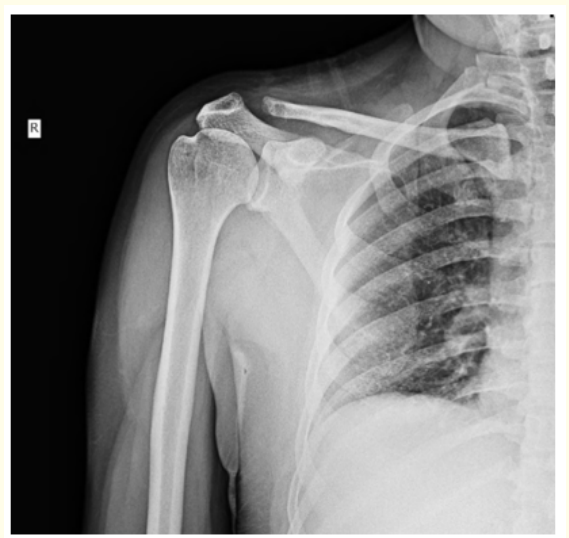

Figure 4: Post- operative X-ray at two weeks.

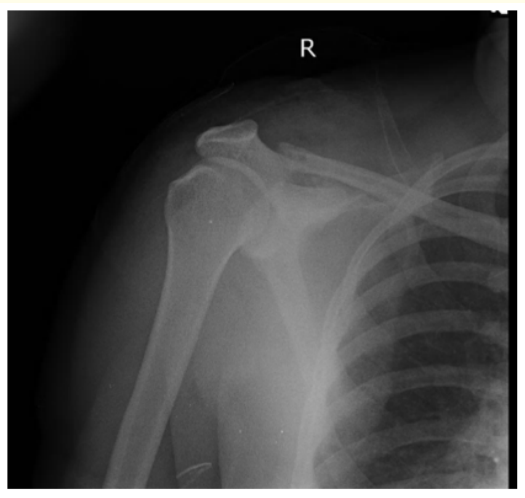

Figure 5: Follow up x-ray at 12 weeks showing restored anatomy. 


\section{Discussion}

AC joint type 1 injury is due to incomplete tear of acromioclavicular ligament, type 2 injury is due to complete disruption of acromioclavicular ligament with intact coracoclavicular ligament, with subluxation of AC joint, and type 3 injury is due to disruption of both acromioclavicular and coracoclavicular ligament with dislocation of $\mathrm{AC}$ joint and superior migration of clavicle or inferior migration of scapula [5]. Type 1 and 2 injuries are mostly managed conservatively. Type 3 injury can be treated conservatively in elderly persons, but requires reconstruction of ligaments in young persons and sportsmen for cosmesis as well as gain of shoulder movement and power. Conservative treatment fails because of interposition of articular disc, frayed capsular ligament and pieces of cartilage lying between the acromion and the clavicle [6].

Various surgical procedures have been described for AC joint dislocation management. By the year 2013, 162 types of different surgical techniques were described for ACJ [7]. Mumford procedure consisting of resection of lateral end of clavicle, is indicated in symptomatic type 2 injury with degenerative arthritis of $\mathrm{AC}$ joint. Neviaser [8] procedure includes reconstruction of superior acromioclavicular ligament with fixation of acromioclavicular joint with Kirschner wire, without any care of coracoclavicular ligament, hence often leads to re-dislocation after outward migration of the wire. Procedures devised for surgical treatment of ACJ dislocation can be grouped as acromioclavicular reduction and fixation; acromioclavicular reduction, coracoclavicular ligament repair and fixation; a combination of the above two procedures; distal clavicle excision; and muscle transfers. Coracoclavicular fixation with heavy non absorbable suture and transfer of the coracoacromial ligament to the distal clavicle has shown $89 \%$ satisfactory result in the study of Weinstein., et al. [6] in early repairs. Coracoclavicular fixation can be done by single or double wire loops, screw, metallic and bioabsorbable suture anchors. Resection of the lateral end of the clavicle is done for both acute and old ACJ dislocation to correct altered ACJ biomechanics. Mazzocca., et al. [4] did anatomic reconstruction of coracoclavicular ligament using autologous semitendinosus graft and augmented it with suture tape, simultaneously doing lateral clavicle excision. Its biomechanical studies showed the fixation to be superior than pin fixation or repair of ligament. Weaver and Dunn [9] used coracoacromial ligament after detaching it from acromion for attaching it to the clavicle after its distal end excision. This procedure was mostly augmented with further fixation in view of incomplete reduction and loss of reduction in follow up.

Tauber., et al. [10] modified Weaver technique by using semitendinosus tendon graft in 12 patients and found that the graft cases had superior clinical and radiological outcome. Arthroscopic transfer of coracoacromial ligament and repair are being done by trained persons, but open surgery gives better field and opportunity to assess and manage associated injuries also. Use of autologous semitendinosus graft or synthetic suture has the advantage of preserving the deltoid insertion [2]. The use of fixation anchors consisting of tendon graft, non- absorbable thick suture material and synthetic tape around the coracoid process reduces the risk of neurovascular injury and decreases surgical time [3].

The combination procedure used in this report consists of, reconstruction of the important anchoring coracoclavicular ligament with the help of non- absorbable thick suture material reinforcing biological strength of autologous semitendinosus tendon graft of the same individual, looped around the coracoid process and passed through a proper size hole in the lateral end of the clavicle, after its oblique osteotomy to make it less prominent. Using the same tendon graft's remaining length for reconstructing acromioclavicular ligament, layer wise closure, stabilization of joint with Kirschner wire, immobilization of shoulder for 6 weeks are other factors helping in good healing of tissue. Gradual active mobilization after 6 weeks healing, gives further time for maturation of connective tissue. The procedure is useful in both acute and old AC joint dislocation or fracture at the lateral end of clavicle presenting like AC dislocation. Additional reinforcement with synthetic tape helps make the loop strong. This procedure is similar to the technique of Mazzocca., et al.

Short term anatomic results of this procedure are satisfying. The technique used is similar to modified Weaver Dunn technique also, except that no attempt was made to use coracoacromial ligament as an anchor to resected clavicle. It requires proper training of graft harvesting, its interlacing with thick suture material, passing carefully around the base of coracoid process and through hole in the resected clavicle and properly tying to have satisfactory long- term outcome. However, the procedure requires further use on many patients and longer follow up to know about its long- term results. Limitation of the report is small number of cases followed for a short period without any objective functional assessment. 


\section{Conclusion}

Reconstruction of coracoclavicular ligament using autologous semitendinosus graft along with thick non-absorbable suture after resecting lateral end of clavicle gives satisfactory result in acute and old acromioclavicular joint dislocation.

\section{Clinical Message}

Reconstruction of coracoclavicular ligament using autologous semitendinosus tendon graft with thick non absorbable suture fixation combined with oblique osteotomy of distal clavicle should be offered as viable surgical option for satisfactory results in fresh and old AC joint dislocation cases.

\section{Bibliography}

1. Renato LT., et al. "Surgical treatment of acromioclavicular dislocation using the endobutton". Acta Ortopédica Brasileira 25 (2017): 81-84.

2. Wolf EM and Pennington WT. "Arthroscopic reconstruction for acromioclavicular joint dislocation". Arthroscopy 17 (2001): 558-563.

3. Wellmann M., et al. "Minimally invasive coracoclavicular ligament augmentation with a flip button/polydioxanone repair for treatment of total acromioclavicular joint dislocation. Arthroscopy 23 (2007): e1-e5.

4. Mazzocca A., et al. Anatomic coracoclavicular reconstruction surgical technique, Technique manual, Naples, FL, Arthrex (2007).

5. Binoti Sheth., et al. "Shoulder Injuries". Text Book of Orthopaedics. 1st ed. CBS publishers and Distributors Pvt Ltd (2018): 50-51.

6. Anthony A Mascioli. "Acute Dislocations". In: Azar FM, Beaty JH and Canale ST, editors. Campbell's Operative Orthopaedics, 13th ed. Elsevier (2017): 3126-3132.

7. Beitzel K., et al. "Current concepts in the treatment of acromioclavicular joint dislocations". Arthroscopy 29 (2013): 387397.

8. Neviaser JS. "Acromioclavicular dislocation treated by transference of the coracoacromial ligament: a long-term followup in a series of 112 cases". Clinical Orthopaedics and Related Research 58 (1968): 57.
9. Andrew H Crenshaw Jr. "Old Unreduced Dislocations". In: Azar FM, Beaty JH and Canale ST, editors. Campbell's Operative Orthopaedics, 13th ed. Elsevier (2017): 3143-3147.

10. Tauber M., et al. "Semitendinosus tendon graft versus a modified Weaver-Dunn procedure for acromioclavicular joint reconstruction in chronic cases: a prospective comparative study". American Journal of Sports Medicine 37 (2009): 181.

\section{Volume 2 Issue 9 September 2019}

(C) All rights are reserved by NS Dhaniwala., et al. 